\title{
Spinal cord pathways
}

\author{
M Dlamini
}

Department of Anaesthesia, Chris Hani Baragwanath Academic Hospital, University of the Witwatersrand, South Africa

Corresponding author, email: cosykhosi@yahoo.com

Keywords: spinal cord pathways, communication, brain, peripheral nervous system

The spinal cord is the primary pathway of communication between the brain and peripheral nervous system. Knowledge of the spinal cord anatomy and recognition of typical common spinal cord syndromes are important as many of these diseases have a predilection for targeting specific areas or tracts within the spinal cord.

\section{Anatomy}

There are 31 spinal cord segments, each with a pair of ventral (anterior) and dorsal (posterior) spinal nerve roots, which mediate motor and sensory function, respectively. The ventral and dorsal nerve roots combine on each side to form the spinal nerves as they exit from the vertebral column through the neuroforamina. ${ }^{1,2}$

\section{Longitudinal organisation}

The spinal cord is divided longitudinally into four regions: cervical (8), thoracic (12), lumbar (5) and sacral cord (5), plus one coccygeal level. It extends from the foramen magnum and terminates near the lower margin of the 1st or 2nd lumbar vertebra. Below that level, the spinal canal contains the lumbar, sacral and coccygeal spinal nerve roots that comprise the cauda equina. Vertebral and spinal cord segmental levels are not necessarily the same because the spinal cord is shorter than the vertebral column. ${ }^{1,2}$

The cervical 1 (C1) through C8 spinal cord segments lie between the $\mathrm{C} 1$ through $\mathrm{C} 7$ vertebral levels. The thoracic 1 (T1) through $\mathrm{T} 12$ cord segments lie between $\mathrm{T} 1$ through T8. The five lumbar ( $\mathrm{L}$ ) cord segments are situated at the T9 through $\mathrm{T} 11$ vertebral levels, and the sacral cord 1 (S1) through S5 segments lie between $\mathrm{T} 12$ and L1 vertebral levels. It is significant to note that the nerve roots for $\mathrm{C} 1$ through $\mathrm{C} 7$ emerge above their respectively numbered vertebrae, and C8 nerve root emerges between $C 7$ and $\mathrm{T} 1$ vertebral bodies., ${ }^{1,2}$ The remaining nerve roots emerge caudal to their respectively numbered vertebrae (Figure 1).

\section{Cervical cord}

The first cervical vertebra (the atlas) and the second cervical vertebra (the axis) support the head at the atlanto-occiput junction. The interface between these is called the atlantoaxis junction. The cervical spinal segments innervate the skin and musculature of the upper extremity and diaphragm. The diaphragm is innervated by the phrenic nerve ( $C 3$ to $C 5)$.

\section{Thoracic cord}

The thoracic segments are identified by having a rib attached to them.

The spinal roots form the intercostal nerves that run along the inferior rib margin and innervate associated dermatomes, as well as the intercostal abdominal wall musculature. ${ }^{1}$

\section{Lumbosacral cord}

It contains segments that innervate the muscles and dermatomes of the lower extremity, as well as the buttocks and anal regions. Sacral nerve roots S3 through S5 originate in the narrow terminal part of the cord, the conus medullaris., ${ }^{1,2}$

\section{Cauda equina}

In adults, the spinal cord ends at the level of the first or second lumbar vertebral bodies. The filum terminale, a thin connective tissue filament that descends from the conus medullaris with the spinal nerve roots, is connected to S3, S4, S5 vertebrae and its terminal part is fused to the periosteum at the base of the coccygeal bone. ${ }^{1,2}$

\section{Cross sectional anatomy}

The spinal cord contains the grey matter, the butterfly shaped central region, and the surrounding white matter tracts. ${ }^{1,2}$

\section{Grey matter}

The spinal cord grey matter, which contains neuronal cell bodies, is made up of the dorsal and ventral horns, each divided into several laminae (Figure 2). ${ }^{1,2}$

\section{Dorsal horn}

It is divided into six layers or laminae that process sensory information. More than that the dorsal horn also modulates pain transmission through spinal and regulatory circuits. There are three major categories of sensory input: 


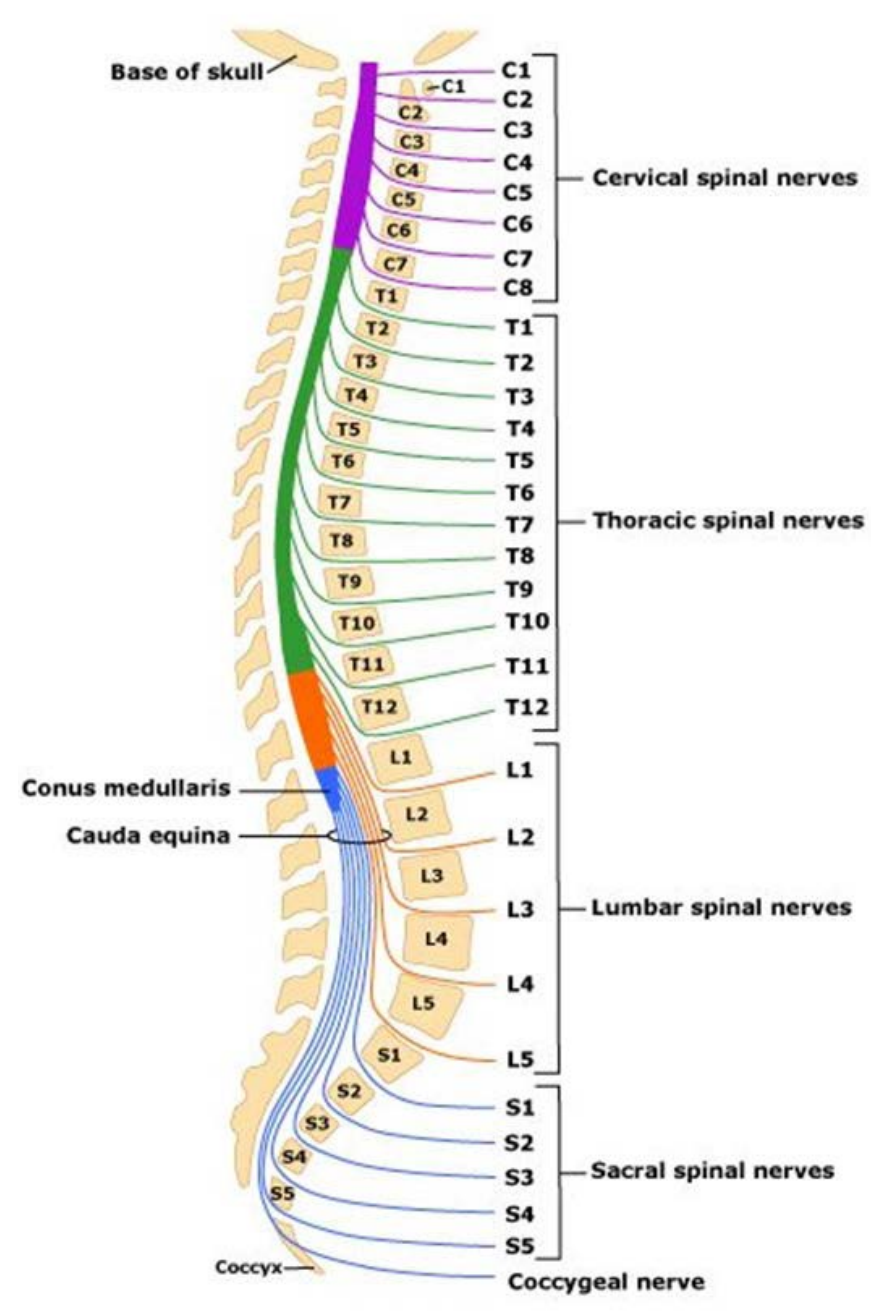

Figure 1: Longitudinal organisation of the spinal cord ${ }^{3}$

- Afferents from muscle spindles that participate in spinal cord reflexes.

- Axons, mostly small unmyelinated, mediating sensory modalities of pain and temperature. These can travel up and down a few segments before synapsing with the second order neurons which cross the midline of the cord in the anterior commissure, and then enter the contralateral anterior or lateral spinothalamic tract.

- Axons mediating the sensory modalities of proprioception, vibration and touch discrimination. These large myelinated fibres pass through the dorsal horn to enter the ipsilateral dorsal column. ${ }^{1,2,4}$

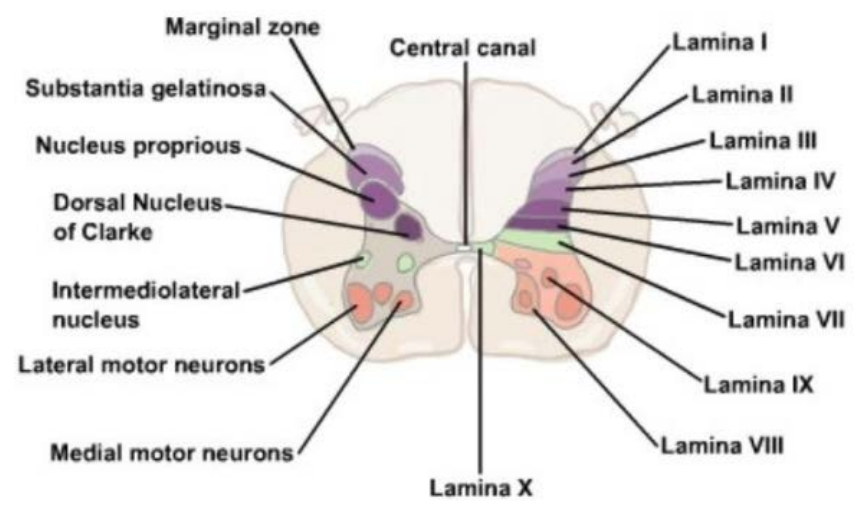

Figure 2: Cross sectional anatomy of the spinal cord ${ }^{6}$

\section{Ventral horn}

The motor nuclei of the spinal cord are contained within the ventral horn, which also contains interneurons mediating information from other descending tracts of the pyramidal and extrapyramidal motor systems. These ultimately synapse on the alpha and gamma motor neurons which subsequently leave the ventral horn via the ventral nerve root to terminate at the neuromuscular junction. . $^{1,45}$

\section{White matter (Figure 3)}

\section{The dorsal or posterior columns}

The fasciculus gracilis, and the fasciculus cuneatus. These contain sensory information regarding joint position and vibration. They are organised anatomically such that cervical sections lie most laterally and sacral segments most medially. These pathways will cross in the medulla, hence in the spinal cord, these tracts contain ipsilateral sensory representation. ${ }^{1,45}$

\section{The anterior and lateral spinothalamic tracts}

These contain sensory information regarding pain, temperature, and touch. These axons have crossed in the ventral commissure and therefore contain contralateral sensory representation. This tract is somatotopically organised with cervical inputs located most medially and sacral inputs most laterally. ${ }^{1,4,5}$

\section{The corticospinal tract (CST)}

This contains the motor neurons that mediate cortical control of bulbar and spinal cord activity. These axons synapse either directly or indirectly on the anterior horn cells, as well as the dorsal spinal cord, traditionally viewed as the "sensory horn." Cortical motoneuronal synapses are likely widely distributed onto many anterior horn cells, allowing for coordination of highly skilled movements. The numeric relationship between cortical motoneurons, their axons, and anterior horn cells is not one-to-one. Each anterior horn cell receives input from many corticomotoneurons (convergence), and a single corticomotorneuron innervates many different anterior horn cells of the same, agonist and antagonist, motor neuron pools (divergence). $2,4,5,7$

The lateral CST - This contains the majority (80-85\%) of these fibres, which have previously decussated (crossed) at the cervicomedullary junction and therefore provide input to the ipsilateral musculature.

The anterior CST -These contain undecussated fibres, some of which will subsequently cross at the spinal level through the anterior commissure.

\section{Other ascending and descending tracts}

The tectospinal tract: This originates in the superior colliculus and mediates reflex postural movements of the head in response to visual and/or acoustic input.

The rubrospinal tract: It originates from the magnocellular subdivision of the red nucleus; is markedly developed in 


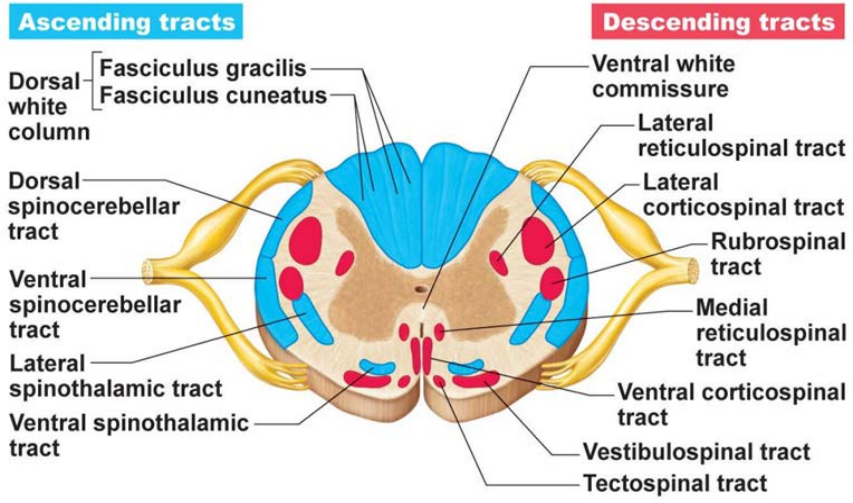

Figure 3: White matter spinal tracts ${ }^{8}$

reptiles, birds, and other lower mammals; but is much less evident in primates, in which there are direct connections with motoneurons innervating wrist muscles.

The vestibulospinal tracts: They arise from the vestibular nuclei and facilitate spinal cord reflexes and muscle tone to maintain posture.
Reticulospinal tracts: They are widely assumed to be responsible for coordinated gross movements primarily of proximal muscles, whereas the CST mediates fine movements, particularly of the hand. However, the reticulospinal system may form a parallel pathway to distal muscles, alongside the CST. As a result, reticulospinal neurons may influence upper limb muscle activity after damage to the corticospinal system, as may occur in stroke.

The dorsal and ventral spinocerebellar tracts: They carry inputs mediating unconscious proprioception directly to the cerebellum. ${ }^{2,45,7}$

The spinoreticular tract: It carries deep pain input to the reticular formation of the brainstem.

\section{Autonomic fibres}

These synapse with cell bodies in the intermediolateral columns of the central grey matter of the spinal cord. Sympathetic fibres exit between $T 1$ and $L 2$, and parasympathetic fibres exit between S2 and S4.
PARASYMPATHETIC NERVES

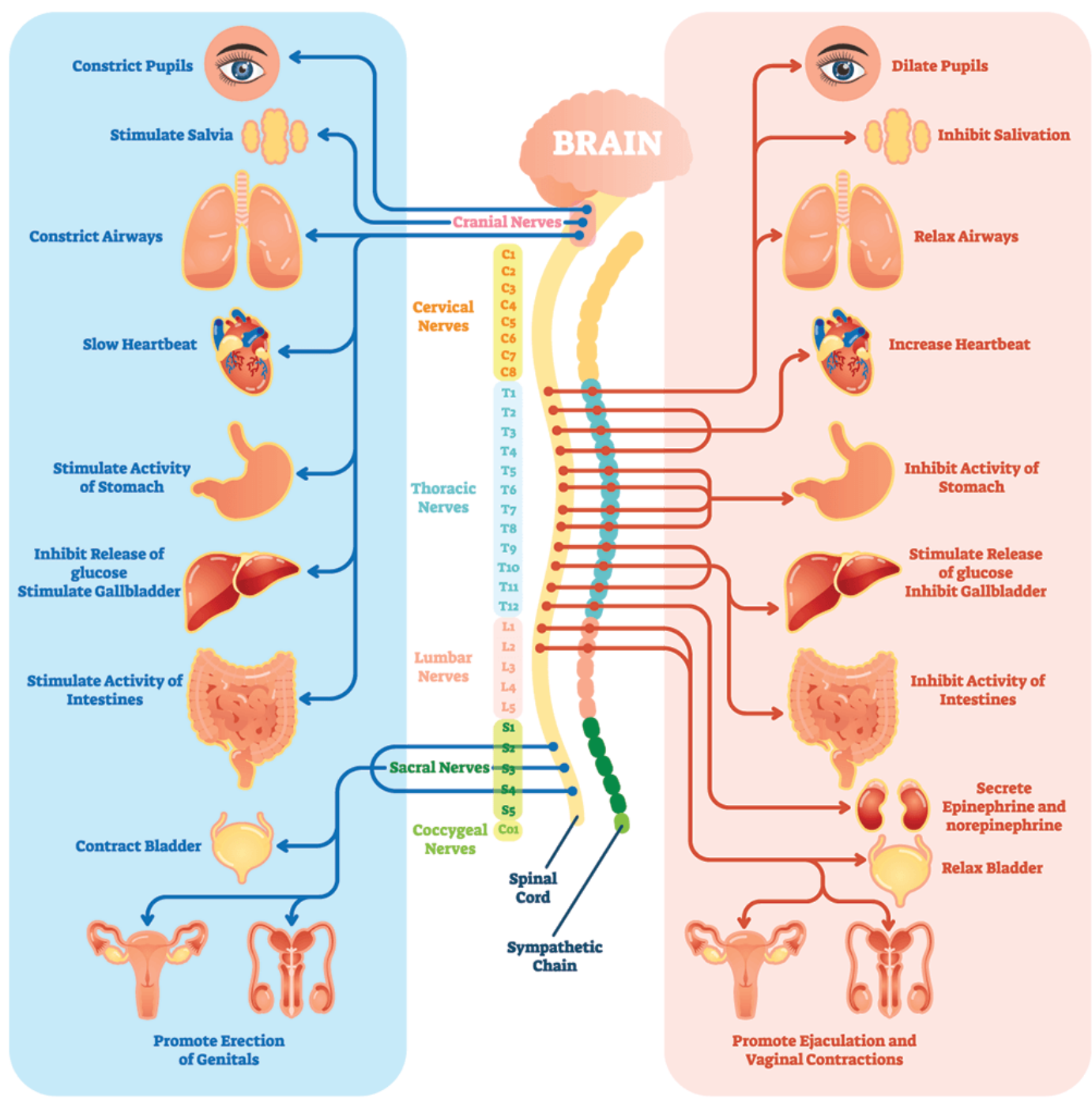

Figure 4: Sympathetic and parasympathetic nervous systems ${ }^{11}$ 
The sympathetic neurons lie in the lateral horn of the central grey matter at spinal levels T1-L3. The preganglionic fibres exit via the ventral root, spinal nerve, and ventral ramus to reach the paravertebral ganglion.

Parasympathetic neurons originate in the sacral spinal cord and exit the spinal cord with other efferents to the ventral ramus. After leaving the ventral ramus, they may subsequently join with sympathetic nerves to reach the viscera. These preganglionic fibres then synapse with a diffuse network of terminal ganglion cells that affect organs in the pelvis (Figure 4).

Autonomic dysfunction is an important determinant of site, extent, and severity of spinal cord pathology. Many autonomic functions can be affected by spinal cord pathology, but for clinical evaluation, the most useful symptoms relate to bladder control. 9,10

Autonomic bladder control is primarily parasympathetic and is unaffected by isolated injury to the sympathetic fibres. Voluntary bladder control is under somatomotor control, mediated by motor fibres originating from the anterior horn cells at levels S2-S4. A spinal cord lesion that interrupts descending motor and autonomic tracts above the S2 level produces an "automatic bladder" that cannot be emptied voluntarily, but empties reflexly when expanded to a certain degree, the so-called neurogenic bladder. Loss of descending inhibition of segmental reflex control leads to urinary urgency and incontinence. Injury to S2-S4 spinal levels interrupts the bladder reflex circuit; the bladder becomes flaccid and fills beyond capacity with overflow incontinence.

Spinal cord transections interrupt voluntary control of the external sphincter and produce constipation. Sacral lesions cause a loss of the anal reflex and rectal incontinence. Impotence can result from spinal cord lesions at any level. Spinal cord injuries can also affect cardiovascular function, most dramatically with lesions above T6, which can produce a phenomenon of autonomic dysreflexia. ${ }^{9,10}$

\section{Pain pathways}

Pain receptors are found on free nerve endings located in many tissues throughout the body. They warn of potentially harmful changes in the environment, such as excessive pressure or temperature. Pain receptors are activated in response to a painful stimulus, usually involving tissue damage. Any kind of stimulus, if intense enough, can stimulate pain receptors in the skin and the mucosa, but only radical changes in the pressure and certain chemicals can stimulate pain receptors in the viscera. ${ }^{7}$ There are three components (Figure 5):

- The first-order neuron transmits pain from the peripheral receptor to the dorsal root ganglion (in which its cell body is found), where it synapses with the...

- second-order neuron found in the dorsal horn of the cord. Its axon crosses the midline and ascends in the spinothalamic tract to the thalamus where it synapses with the...

- third-order neuron, which projects to the postcentral gyrus..$^{1,5,7}$

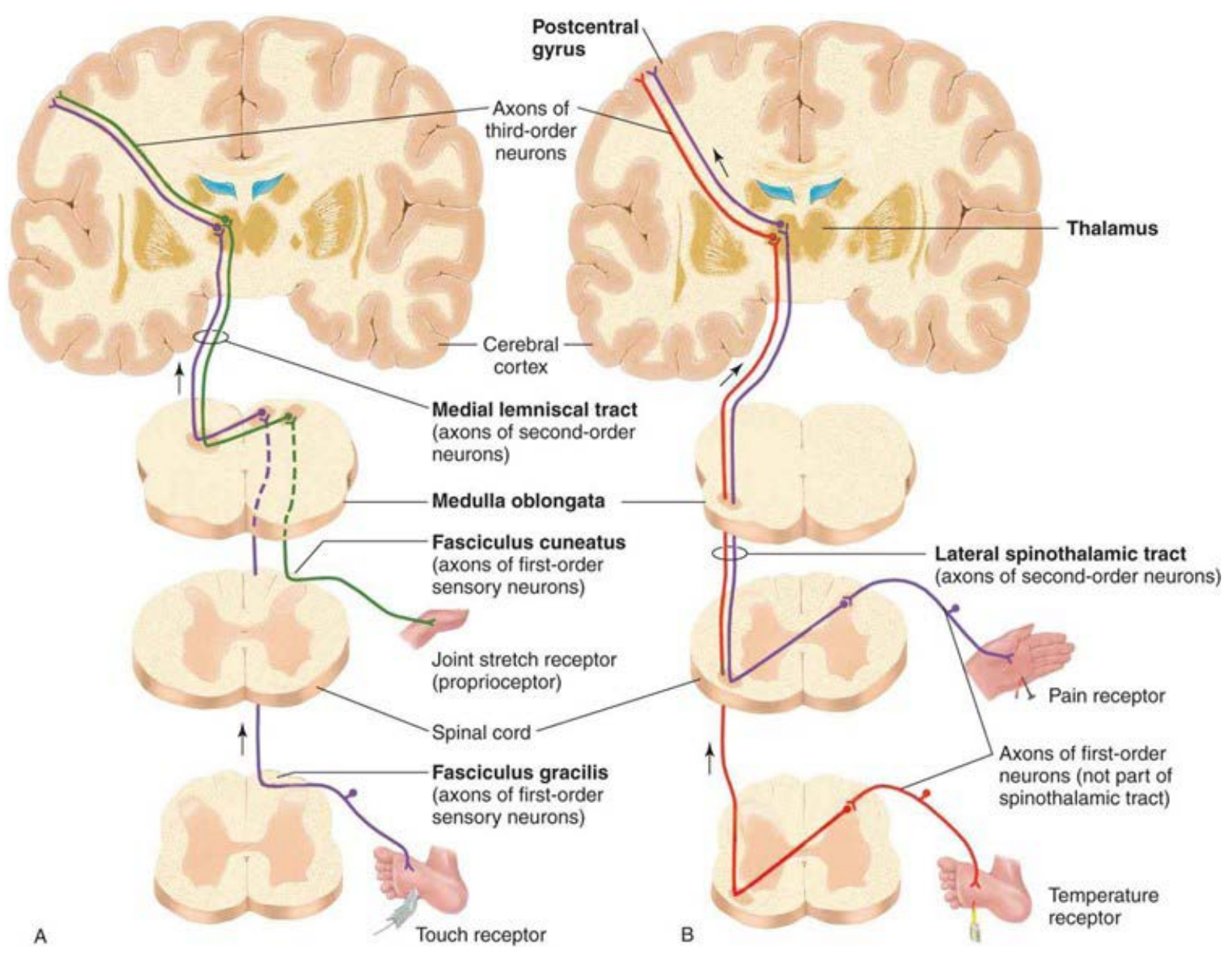

Figure 5: Position, pain and pressure pathway ${ }^{12}$ 
Table I: Spinal cord syndromes $\mathbf{1}^{1,2,5,14}$

\begin{tabular}{ll}
\hline Syndrome & Clinical picture \\
\hline Segmental (transection) syndrome & Loss of all sensory modalities, weakness below affected level, bladder dysfunction \\
\hline Dorsal cord syndrome & Loss of proprioception, vibratory sensation, variable weakness and bladder dysfunction \\
\hline Ventral cord syndrome (anterior spinal artery syndrome) & Loss of pain and temperature sensation, weakness, bladder dysfunction \\
\hline Brown-Séquard syndrome & $\begin{array}{l}\text { Ipsilateral weakness and loss of proprioception, contralateral loss of pain and } \\
\text { temperature sensation }\end{array}$ \\
\hline Central cord syndrome & Segmental loss of pain and temperature, weakness often greater in arms than legs \\
\hline Pure motor syndrome & Weakness without sensory disturbance \\
\hline Conus medullaris syndrome & Bladder and rectal dysfunction, saddle anaesthesia \\
\hline Cauda equina syndrome & Asymmetric multi radicular pain, leg weakness, and sensory loss, bladder dysfunction \\
\hline
\end{tabular}

\section{Blood supply to the spinal cord}

A single anterior and two posterior spinal arteries supply the spinal cord. The anterior spinal artery supplies the anterior twothirds of the cord. The posterior spinal arteries primarily supply the dorsal columns. The anterior and posterior spinal arteries arise from the vertebral arteries in the neck and descend from the base of the skull. Various radicular arteries branch off the thoracic and abdominal aorta to provide additional blood supply to the spinal arteries.

The largest and most consistently present of these radicular branches is the great ventral radicular artery or the artery of Adamkiewicz, which supplies the anterior spinal artery. This artery enters the spinal cord anywhere between T5 and L1, usually between T9 and T12. ${ }^{1,5,7}$ The primary watershed area of the spinal cord in most people is in the midthoracic region.

The spinal cord drains via the single anterior spinal vein and single posterior spinal vein, which in turn drain into the internal vertebral venous plexus located in the epidural space within the extradural fat. These veins eventually empty into the external venous plexus, and via the basivertebral veins..$^{1,5,7}$

\section{Clinical significance}

A spinal cord lesion may be suspected when there are bilateral motor and sensory signs or symptoms that do not involve the head or face. Motor deficits are manifest by weakness and long tract signs (spasticity, increased reflexes, Babinski sign). When the pathology is localised or segmental, these findings will be present in muscle groups innervated below that level and will be normal above. A sensory level, with normal sensation above and reduced or absent below, can also often be defined and should be specifically sought. Some disorders affecting the spinal cord preferentially affect different structures, and therefore careful testing of all spinal cord functions, including motor, reflex, and all sensory modalities, and sphincter function is important for clinical localisation. ${ }^{1,4,13}$

Several distinct spinal cord syndromes are recognised (Table I).

\section{Conflict of interest}

The author declares no conflict of interest.

\section{Funding source}

None.

\section{ORCID}

M Dlamini (iD https://orcid.org/0000-0002-2834-6279

\section{References}

1. Haines DE, Mihailoff GA, Yierski RP. The spinal cord. In: Haines DE, Mihailoff GA editors. Fundamental neuroscience for basic and clinical applications. 5th ed. Philadelphia: Elsevier Inc. ; 2018. p138-51. e1.

2. Crossman AR, Neary D. Neuroanatomy E-book: an illustrated colour text. 6th ed.Elsevier Health Sciences; 2014.

3. ongitudinal organization of spinal cord, spinal nerves, and vertebrae [Internet] UpTodate. Longitudinal Organization; 2011. Available from: https://somepomed. org/articulos/contents/mobipreview.htm?21/34/22050.

4. Jones Jr HR, Burns T, Aminoff MJ, Pomeroy S. The Netter collection of medical illustrations: nervous system, Volume 7, Part II-Spinal cord and peripheral motor and sensory systems. E-book. Elsevier Health Sciences; 2013.

5. Costanzo LS. Neurophysiology. Physiology. 6th ed. Philadelphia: Elsevier; 2018. p. 69-116.

6. Lunatic Laboratories [Internet]. Day 164: Know your spinal cord - The grey matter; Cross sectional anatomy of the spinal cord. c2020. Available from: https://loonylabs.files.wordpress.com/2020/01/spinal-organization.jpg?w=590.

7. Westlund KN. Pain Pathways: Peripheral, spinal, ascending, and descending pathways. Practical management of pain: Elsevier; 2014. p. 87-98. e5. https://doi. org/10.1016/B978-0-323-08340-9.00008-6.

8. White matter spinal tracts: Koibana.info; 2020. Available from: https://i.pinimg. com/originals/6a/c5/da/6ac5da7aeb3e4c27d9822b237f18681a.jpg.

9. Mtui E, Gruener G, Dockery P. Fitzgerald's clinical neuroanatomy and neuroscience. E-book. Elsevier; 2020.

10. Wang S, Fehlings MG. Medical management of spinal cord injury. In: Winn HR, editor. Youmans and Winn neurological surgery. 7th ed. Philadelphia: Elsevier Inc.; 2017. p. 2493-504.e3.

11. Parasympathetic and sympathetic nerves. Available from: https://www. sci-infopages.com/wp-content/media/autonomic-nerve-pathways-768×803. png.

12. Position, pressure, pain pathway. Available from: https://doctorlib.info/ physiology/review/review.files/image227.jpg.

13. Biglioli $P$, Roberto $M$, Cannata $A$, et al. Upper and lower spinal cord blood supply: the continuity of the anterior spinal artery and the relevance of the lumbar arteries. J Thorac Cardiovasc Surg. 2004;127(4):1188-92. https://doi. org/10.1016/j.jtcvs.2003.11.038.

14. Seecharan DJ, Arnold PM. Spinal cord injuries and syndromes. In: Shen FH Samartzis D, Fessler RG, editors. Textbook of the cervical spine. Missouri: Saunders, an imprint of Elsevier Inc.; 2015. p. 192-6. 